\title{
Análisis geoestadístico de las barrancas Colorada y Quimichule del volcán Popocatépetl (México) como aporte a la prevención de desastres naturales
}

\author{
Rogelio Ramos-Aguilar¹, rogelio.ramos@correo.buap.mx, Patricia Máximo-Romero' ${ }^{1}$, Blanca \\ Susana Soto-Cruz ${ }^{2}$,Salvador Alcántara-Iniesta ${ }^{2}$ y María de la Cruz Vázquez-García ${ }^{3}$
}

\begin{abstract}
RESUMEN
Este trabajo se presenta como una contribución a la prevención de desastres naturales y para generar un complemento a otros estudios semejantes. El volcán Popocatépetl ha presentado actividad importante y constante desde el año 1994. Dentro de su estructura geológica se localizan las barrancas Colorada y Quimichule, que por sus características topográficas, el material volcánico expulsado y las lluvias torrenciales que se han presentado en los últimos años, ponen en riesgo a las poblaciones cercanas. Se presenta un análisis geoestadístico para obtener la aceleración de la gravedad, la pendiente por distanciaelevación, la altura-gravedad y la fuerza de un líquido sobre las barrancas. La conversión de coordenadas UTM a geográficas se hizo con el programa Traninv aplicando el Datum ITRF2008 y la zona 14, la gravedad local se calculó con el programa Organización Internacional de Metrología Legal (OIML) y el análisis estadístico con el Geoestatistical Enviromenal Assessment. La modelación estructural se realizó con Surfer y se utilizaron modelos hidrológicos para calcular el gasto y la fuerza. Del análisis de correlación se concluyó que la barranca con mayor gravedad es la de Quimichule, la que transportaría con mayor velocidad flujos de lodo con ceniza volcánica. Se aplicaron las técnicas y modelos cartográficos, geomorfológicos y estadísticos acordes al estudio, para obtener los resultados presentes.
\end{abstract}

Palabras clave: desastre natural, geoestadística, fuerza, aceleración, gravedad.

\section{Geostatistical analysis of the Quimichule y Colorada canyons from Popocatépetl (México) volcano as a contribution to the prevention of natural disasters}

\begin{abstract}
This paper aims to contribute to the prevention of natural disasters and generate a complement to other similar studies. The Popocatépetl volcano has showed significant and constant activity since 1994. Within its geologic structure are located Colorada and Quimichule streams, due to its topographic features, volcanic material ejected and torrential rains in last years the mentioned volcano hazards nearby towns. This work shows a geostatistical analysis to obtain the gravity acceleration, slope by the distance-elevation relation, height-gravity and the fluid force on streams. The conversion of UTM coordinates to geographical was made with the use of the program Traninv applying the ITRF2008 Datum and the 14 zone, the local gravity was calculated with the use of International Organization of Legal Metrology (OIML) and the statistical analysis was obtained with the use of Geoestatistical Enviromenal Assessment. The structural modeling was performed with the use of Sufer and to calculate spending and force was used hydrological models. Correlation analysis concluded that Qumichule is the stream with major gravity and also this stream transport faster mudflows with ash. The techniques were applied and mapping, geomorphological and statistical models in accordance with the study to obtain the expected results, the application of other models would probably come to the same conclusions.
\end{abstract}

Keywords: natural disasters, geostatistical, force, acceleration, gravity.

Recibido el 19 de febrero de 2013, aceptado el 18 de julio de 2014.

Facultad de Ingeniería, Benemérita Universidad Autónoma de Puebla. Av. San Claudio y 18 sur, C.U., Puebla, México.

2 Centro de Investigación en Dispositivos Semiconductores. Benemérita Universidad Autónoma de Puebla. Av. San Claudio y 18 sur, C.U., Puebla, México.

3 Bufete de Ingeniería en Telecomunicaciones y Sistemas. México, S. A. de C. V., José Toribio Medina 130. México, D. F. 


\section{INTRODUCCIÓN}

El volcán Popocatépetl se encuentra situado a los $19^{\circ} 17^{\prime}$ de latitud Norte y a los $98^{\circ} 38^{\prime}$ longitud Occidental del meridiano de Greenwich. Tiene una altura de 5.420 m.s.n.m y colinda con los estados de Puebla, Morelos y México. El volcán Popocatépetl inició en 1994 una etapa importante de actividad manifestando expulsión de vapor de agua, ceniza y roca incandescente, y eventos sísmicos. A partir de entonces, se ha mantenido activo con períodos de alta y baja intensidad. La constante actividad, las lluvias torrenciales atípicas causadas por el cambio climático, el deshielo del glaciar existente y la topografía, favorecen los deslizamientos de flujos de lodo con ceniza volcánica en las barrancas como la Colorada y Quimichule, mismos que están relacionados directamente con la gravedad, la pendiente, la altura y el gasto, y que pondrían en riesgo a las comunidades aledañas. Además, los cambios de presión generados por la actividad dentro de la cámara magmática producen deformación y variaciones de gravedad en la superficie del volcán, las que deben cuantificarse periódicamente. Después de la erupción de 1994, se realizó un estudio denominado Posible Flujo de Lodo en el Costado Oriente del Volcán Popocatépetl (GARCÍA et al. 1996). En este estudio se construyó un perfil de la barranca El Aguardientero y se calcularon sus pendientes cada $100 \mathrm{~m}$ de elevación, utilizando cartas topográficas del INSTITUTO NACIONAL DE ESTADÍSTICA Y GEOGRAFÍA a una escala 1:50.000, determinando que una de las zonas con mayor acumulación de ceniza es San Pedro Benito Juárez, por donde pasa la barranca El Aguardientero.

El presente trabajo se plantea como una contribución a la prevención de desastres naturales volcánicos, específicamente a través del desarrollo de metodologías geoestadísticas. Contar con esta información servirá para determinar acciones de prevención en situaciones de actividad volcánica generadas por deshielo cuando exista una actividad volcánica mayor; actualmente se continúan estudios para calcular otras variables geomorfológicas.

Se han realizado estudios de estabilidad en barrancas aplicando métodos geotécnicos y de elementos finitos para determinar tensiones, deformaciones $\mathrm{y}$ resistencias al corte (FRANCO et al. 2009). Estos métodos están basados en modelos matemáticos que proporcionan una aproximación a la solución del problema, pero sin tomar en cuenta los métodos geodésicos para determinar la pendiente y la gravedad. Los métodos geodésicos proporcionan resultados más aproximados a la realidad, pues los datos que se inyectan a los programas de cómputo se obtienen a través de lecturas en cartas topográficas con una buena aproximación.

La localización de este volcán y la actividad que ha presentado en los últimos años, lo identifica como una estructura de riesgo para las comunidades que se encuentran cerca del mismo.

Con relación a este trabajo, no existe información de estudios o investigaciones similares en la zona del Popocatépetl, siendo importante conocer los posibles darían que se presentarían en el caso de una erupción mayor. La figura 1 muestra la topografía del área de estudio.

\section{MATERIALES Y MÉTODOS}

Las coordenadas UTM del perfil de las barrancas se obtuvieron a través de la lectura de cartas topográficas digitales del volcán y que se encuentran a disposición de los usuarios en el sitio 
web del INSTITUTO NACIONAL DE ESTADÍSTICA Y GEOGRAFÍA. Con estas cartas se definieron los puntos donde se calcularon la aceleración de la gravedad, la pendiente por distanciaelevación y la gravedad-altura. Las coordenadas UTM se convirtieron a geográficas con el programa Traninv, aplicando el Datum ITRF2008 y la zona 14. Actualmente, toda la cartografía y productos digitales producidos por el INEGI, utiliza el que entró en vigor en diciembre de 2010 y que establece como marco de referencia oficial al ITRF2008 en época 2010.0 en sustitución del ITRF92 época 1988.0. liberando al mismo tiempo un software (Traninv) basado en el algoritmo matemático para la transformación o conversión de coordenadas de ITRF92 a ITRF2008, con lo cual se busca una transición suave hacia un nuevo sistema de referencia. La aceleración local de la gravedad se calculó con el programa que se encuentra en el sitio web del CENTRO NACIONAL DE METROLOGÍA, basado en el boletín OIML-N 127 de 1992.
El análisis estadístico se realizó con el Geoestatistical Enviromenal Assessment y la modelación estructural con el programa Surfer. Además, se utilizaron modelos hidrológicos para calcular el gasto y la fuerza de un líquido. Posteriormente, se obtuvieron las relaciones entre gravedad-altura $y$ gravedad-pendiente, y los correspondientes coeficientes de correlación.

La correlación entre las tres variables estudiadas es importante porque define el comportamiento de flujos sobre las barrancas, con lo que se tendría una idea de la ubicación de la zona de mayor riesgo en esas estructuras geológicas.

\section{Conversión de coordenadas de los datos registrados}

Para cada barranca los datos se obtuvieron de la carta topográfica E14B42, obteniendo las coordenadas UTM y posteriormente transformándolas a geográficas con el programa Traninv, donde $\mathrm{X}=\mathrm{W}$ e $\mathrm{Y}=\mathrm{N}$ (Fig.2).

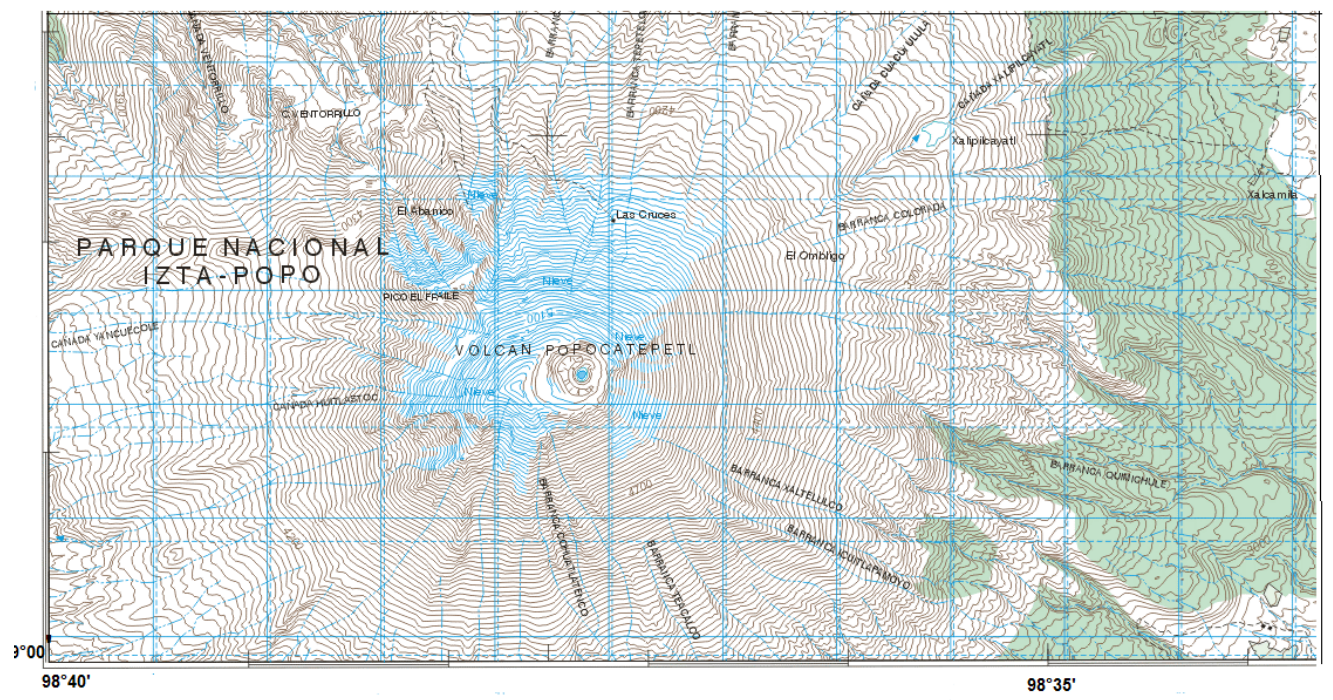

Fig. 1. Topografía del volcán Popocatépetl en el Parque Nacional Izta-Popo, México.

Fig. 1. Topography of Volcán Popocatépetl in the Izta-Popo National Park, México. 


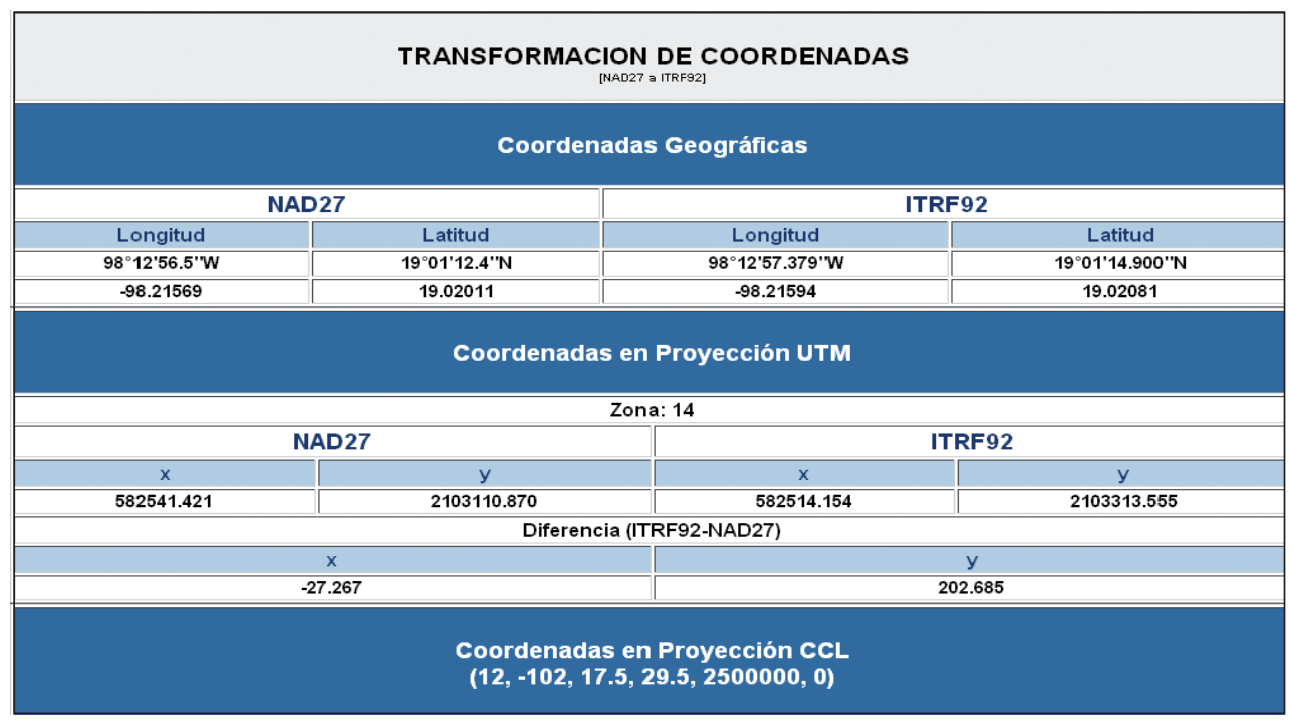

Fig. 2. Modelo de transformación de las coordenadas de UTM a geográficas para todas las lecturas de las barrancas.

En las Tablas 1 y 2 se muestran los datos que fueron utilizados para el procesamiento cada 500 metros de longitud para cada barranca.

Tabla 1. La barranca Colorada tiene una longitud de 6.5 KM, por lo Que Se realizaron 13 LECTURAS ( $Z=$ AltURA, $N=$ LATITUD, $W=$ LONGITUD, EN METROS).

\begin{tabular}{|c|c|c|c|c|c|}
\hline \multicolumn{7}{|c|}{ "Datos" } \\
\hline Punto & Latitud & Longitud & Z & W & N \\
\hline 1 & $19^{\circ} 02^{\circ} 04.2^{\circ}$ & $98^{\circ} 36^{\circ} 09.827^{\circ}$ & 4200 & 541805.29189908 & 2104565.59683705 \\
\hline 2 & $19^{\circ} 02^{\circ} 08.988^{\circ}$ & $98^{\circ} 35^{\circ} 53.520^{\circ}$ & 4055 & 542281.71412205 & 2104714.52382324 \\
\hline 3 & $19^{\circ} 02^{\circ} 11.112^{\circ}$ & $98^{\circ} 35^{\circ} 36.960^{\circ}$ & 3940 & 542765.33555756 & 2104780.79813034 \\
\hline 4 & $19^{\circ} 02^{\circ} 15.864^{\circ}$ & $98^{\circ} 35^{\circ} 24.18^{\circ}$ & 3800 & 543138.27430897 & 2104927.04944176 \\
\hline 5 & $19^{\circ} 02^{\circ} 17.303^{\circ}$ & $98^{\circ} 35^{\circ} 06.324^{\circ}$ & 3640 & 543659.93448173 & 2104973.45727285 \\
\hline 6 & $19^{\circ} 02^{\circ} 19.428^{\circ}$ & $98^{\circ} 34^{\circ} 49.439^{\circ}$ & 3440 & 544153.19176827 & 2105039.48355822 \\
\hline 7 & $19^{\circ} 02^{\circ} 19.428^{\circ}$ & $98^{\circ} 34^{\circ} 32.375^{\circ}$ & 3315 & 544652.15627396 & 2105040.68221700 \\
\hline 8 & $19^{\circ} 02^{\circ} 12.336^{\circ}$ & $98^{\circ} 34^{\circ} 15.311^{\circ}$ & 3195 & 545151.65408519 & 2104823.66422687 \\
\hline 9 & $19^{\circ} 02^{\circ} 11.220^{\circ}$ & $98^{\circ} 33^{\circ} 58.247^{\circ}$ & 3065 & 545650.70770577 & 2104791.38677296 \\
\hline 10 & $19^{\circ} 02^{\circ} 06.072^{\circ}$ & $98^{\circ} 33^{\circ} 41.183^{\circ}$ & 2985 & 546149.78489721 & 2104633.40908685 \\
\hline 11 & $19^{\circ} 02^{\circ} 00.168^{\circ}$ & $98^{\circ} 33^{\circ} 24.120^{\circ}$ & 2920 & 546649.21750056 & 2104453.92978774 \\
\hline 12 & $19^{\circ} 01^{\circ} 49.619^{\circ}$ & $98^{\circ} 33^{\circ} 07.056^{\circ}$ & 2855 & 547149.02607009 & 2104130.92352549 \\
\hline 13 & $19^{\circ} 01^{\circ} 48.107^{\circ}$ & $98^{\circ} 33^{\circ} 05.22^{\circ}$ & 2840 & 547202.93224521 & 2104084.34099710 \\
\hline
\end{tabular}




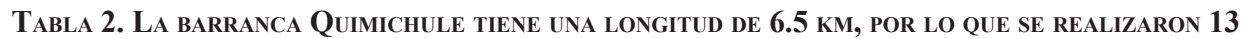

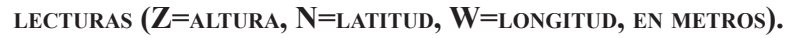

\begin{tabular}{|c|c|c|c|r|r|}
\hline \multicolumn{7}{|c|}{ "Datos" } \\
\hline Punto & Latitud & Longitud & $\mathbf{Z}$ & $\mathbf{~ W}$ & $\mathbf{~ N}$ \\
\hline 1 & $19^{\circ} 01^{\circ} 17.760$ & $98^{\circ} 35^{\circ} 49.847^{\circ}$ & 3920 & 542391.91 & 2103140.27 \\
\hline 2 & $19^{\circ} 01^{\circ} 09.012$ & $98^{\circ} 35^{\circ} 36.060^{\circ}$ & 3680 & 542795.823 & 2102872.32 \\
\hline 3 & $19^{\circ} 01^{\circ} 00.768$ & $98^{\circ} 35^{\circ} 21.803^{\circ}$ & 3500 & 543213.32 & 2102619.41 \\
\hline 4 & $19^{\circ} 00^{\circ} 58.68$ & $98^{\circ} 35^{\circ} 05.8566^{\circ}$ & 3360 & 543679.56 & 2102556.7 \\
\hline 5 & $19^{\circ} 00^{\circ} 56.484$ & $98^{\circ} 34^{\circ} 49.008^{\circ}$ & 3250 & 544172.501 & 2102490.61 \\
\hline 6 & $19^{\circ} 00^{\circ} 54.432$ & $98^{\circ} 34^{\circ} 32.808^{\circ}$ & 3150 & 544645.769 & 2102428.8 \\
\hline 7 & $19^{\circ} 00^{\circ} 51.731$ & $98^{\circ} 34^{\circ} 16.32^{\circ}$ & 3080 & 545128.049 & 2102346.38 \\
\hline 8 & $19^{\circ} 00^{\circ} 52.020$ & $98^{\circ} 34^{\circ} 01.050^{\circ}$ & 3030 & 545574.45 & 2102356.31 \\
\hline 9 & $19^{\circ} 00^{\circ} 30.183$ & $98^{\circ} 33^{\circ} 46.368^{\circ}$ & 2990 & 546003.96 & 2102300.98 \\
\hline 10 & $19^{\circ} 00^{\circ} 36.359$ & $98^{\circ} 33^{\circ} 40.572^{\circ}$ & 2900 & 546174.736 & 2101876.96 \\
\hline 11 & $19^{\circ} 00^{\circ} 25.128$ & $98^{\circ} 33^{\circ} 30.600^{\circ}$ & 2840 & 546466.746 & 2101532.29 \\
\hline 12 & $19^{\circ} 00^{\circ} 11.34$ & $98^{\circ} 33^{\circ} 23.507^{\circ}$ & 2780 & 546675.861 & 2101108.85 \\
\hline 13 & $18^{\circ} 59^{\circ} 59.856$ & $98^{\circ} 33^{\circ} 14.148^{\circ}$ & 2730 & 546949.951 & 2100756.32 \\
\hline
\end{tabular}

Desarrollo para el cálculo de la gravedad local

La aceleración de la gravedad es la manifestación de la atracción universal que impulsa los cuerpos hacia el centro de la Tierra, es fuerza que determina el peso de los cuerpos (FRANCO et al. 2005). La aceleración de la gravedad se denota por $g$ y se define como el incremento constante de la velocidad por unidad de tiempo, percibido por un cuerpo en caída libre, es inversamente proporcional a la masa $m_{0}$ del cuerpo en kilogramos $(\mathrm{kg}): g=F / m_{0}$. Para calcular la gravedad local de cada barranca se aplicó el programa recomendado por la Organización Internacional de Metrología Legal (OIML) (Fig. 3).

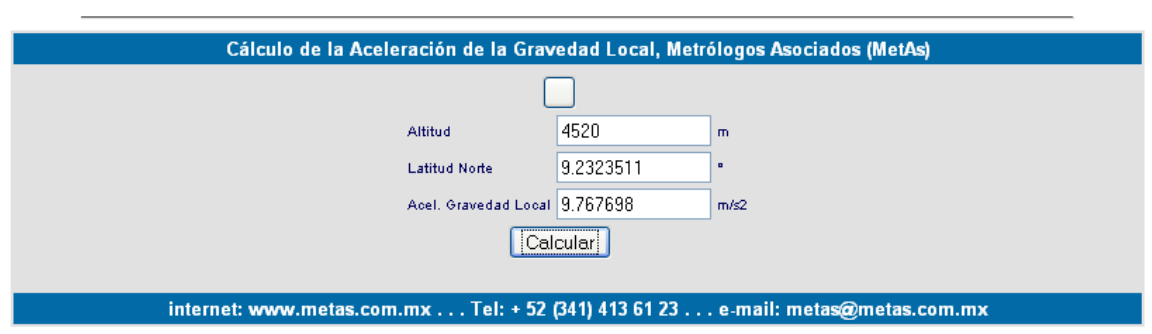

Fig. 3. Ejemplo del cálculo de la aceleración de la gravedad local.

Para corroborar los resultados de la gravedad en diferentes puntos de la barranca cada 500 metros de distancia, se utilizó la ecuación (1), misma que puede calcularse con una exactitud del orden $0.001 \%=100 \mathrm{ppm}$. Los datos que se introducen son: altitud (m) y latitud norte $\left({ }^{\circ}\right)$. Este programa calcula la aceleración local de la gravedad aplicando la ecuación (1) (Tablas 3 y 4) 
$\mathrm{gl}=\left[\mathrm{ge}^{*}\left(1+\left(\mathrm{f}^{\mathrm{*} *} \operatorname{sen}^{2} \phi\right)-\left(\mathrm{F}_{4}{ }^{*} \operatorname{sen}^{2} 2 \phi\right)\right)\right]-\left(\mathrm{Dg}^{*} \mathrm{~h}\right)$

Donde:

$\mathrm{gl}=$ Aceleración de la gravedad local $\left(\mathrm{m} / \mathrm{s}^{2}\right)$.

ge $=9.7803185 \mathrm{~m} / \mathrm{s}^{2}$, aceleración de la gravedad en el Ecuador $(\varphi=0)$.

$\mathrm{f}^{\prime}=0.0053024$ (aplastamiento gravitacional).

$\phi=$ Latitud en grados, minutos, segundos ( $00^{\circ} 00$ ' $\left.00^{\prime \prime}\right)$.

$\mathrm{h}=$ Altitud sobre el nivel medio del mar (m).

$\mathrm{F}_{4}=0.0000059$

$\mathrm{Dg}=0.000003086$

Tabla 3. Barranca Colorada. Se calculó la gravedad cada 500 metros, obteniéndose el PROMEDIO DE $9.775309 \mathrm{M} / \mathrm{s}^{2}$.

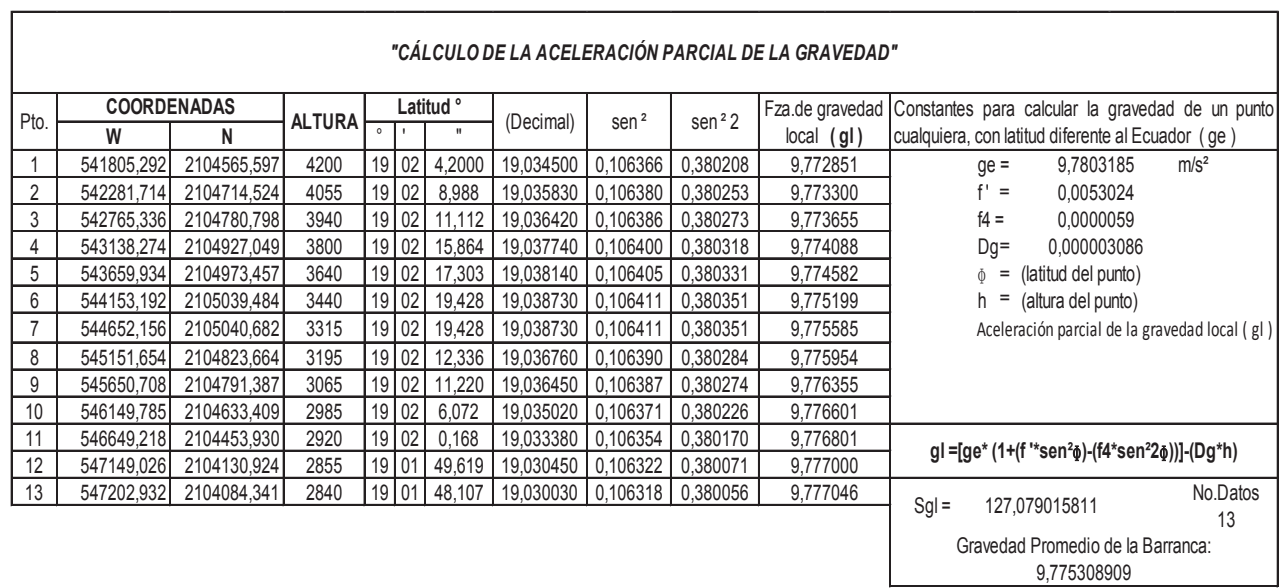

Tabla 4. Barranca Quimichule. Se calculó la GRavedad Cada 500 metros obTeniéndose el PROMEDIO DE 9.776018

\begin{tabular}{|c|c|c|c|c|c|c|c|c|c|c|c|}
\hline \multicolumn{12}{|c|}{ "CÁLCULO DE LA ACELERACIÓN PARCIAL DE LA GRAVEDAD" } \\
\hline Pto. & \multicolumn{2}{|c|}{ COORDENADAS } & ALTURA & \multicolumn{3}{|c|}{ Latitud $^{\circ}$} & (Decimal) & $\operatorname{sen}^{2}$ & $\operatorname{sen}^{2} 2$ & $\begin{array}{c}\begin{array}{c}\text { Fza.de gravedad } \\
\text { local }(\mathrm{gl})\end{array} \\
\end{array}$ & $\begin{array}{l}\text { Constantes para calcular la gravedad de un punto } \\
\text { cualquiera, con latitud diferente al Ecuador ( ge ) }\end{array}$ \\
\hline 1 & $\frac{W}{541805,292}$ & $\frac{N}{2104565,597}$ & 3920 & 19 & 01 & 4,200 & 19,017833 & 0,106186 & 0.379643 & 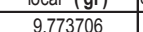 & \multirow{10}{*}{$\begin{array}{|lc|}\mathrm{ge}= & 9,7803185 \\
\mathrm{f}^{\prime}= & 0,0053024 \\
\mathrm{f} 4= & 0,0000059 \\
\mathrm{Dg} & =0,000003086 \\
\Phi & =\text { (latitud del punto) } \\
\mathrm{h}= & \text { (altura del punto) } \\
\text { Aceleración parcial de la gravedad local }(\mathrm{gl})\end{array}$} \\
\hline 2 & 542281,714 & 2104714,524 & 3680 & 19 & 01 & 8,988 & 19,019163 & 0,106201 & 0,379688 & 9,774448 & \\
\hline 3 & 542765,336 & 2104780,798 & 3500 & 19 & 01 & 0,768 & 19,016880 & 0,106176 & 0,379611 & 9,775002 & \\
\hline 4 & 543138,274 & 2104927,049 & 3360 & 19 & 00 & 58,680 & 19,016300 & 0,106170 & 0,379591 & 9,775434 & \\
\hline 5 & 543659,934 & 2104973,457 & 3250 & 19 & \begin{tabular}{l|}
00 \\
\end{tabular} & \begin{tabular}{|l|}
56,484 \\
\end{tabular} & 19,015690 & 0,106163 & 0,379571 & 9,775773 & \\
\hline 6 & 544153,192 & 2105039,484 & 3150 & 19 & 00 & 54,432 & 19,015120 & 0,106157 & 0,379551 & 9,776081 & \\
\hline 7 & 544652,156 & 2105040,682 & 3080 & 19 & 00 & 51,731 & 19,014370 & 0,106149 & 0,379526 & 9,776297 & \\
\hline 8 & 545151,654 & 2104823,664 & 3030 & 19 & 00 & 52,020 & 19,014450 & 0,106150 & 0,379529 & 9,776451 & \\
\hline 9 & 545650,708 & 2104791,387 & 2990 & 19 & \begin{tabular}{|l|}
00 \\
\end{tabular} & 50,183 & 19,013940 & 0,106144 & 0,379511 & 9,776574 & \\
\hline 10 & 546149,785 & 2104633,409 & 2900 & 19 & 00 & 36,359 & 19,010100 & 0,106103 & 0,379381 & 9,776850 & \\
\hline 11 & 546649,218 & 2104453,930 & 2840 & 19 & 00 & 25,128 & 19,006980 & 0,106070 & 0,379275 & 9,777033 & \multirow{2}{*}{$g \mid=\left[g e^{*}\left(1+\left(f^{\prime *} \operatorname{sen}^{2} \Phi\right)-\left(f 4^{*} \operatorname{sen}^{2} 2 \Phi\right)\right)\right]-\left(D^{*} h\right)$} \\
\hline 12 & 547149,026 & 2104130,924 & 2780 & 19 & 00 & 11,340 & 19,003150 & 0,106028 & 0,379146 & 9,777216 & \\
\hline 13 & 547202,932 & 2104084,341 & 2730 & 18 & 59 & 59,856 & 18,999960 & 0,105994 & 0,379038 & 9,777369 & \multirow{2}{*}{$\begin{array}{cc}\text { Sgl }= & \begin{array}{c}\text { No.Datos } \\
127,088231465\end{array} \\
\text { Gravedad Promedio de la Barranca: } \\
9,776017805\end{array}$} \\
\hline & & & & & & & & & & & \\
\hline
\end{tabular}




\section{Cálculo de la pendiente}

La pendiente es la relación que existe entre el desnivel y la distancia en horizontal al plano, lo que equivale a la tangente del ángulo que forma la línea a medir con el eje X. Ver ecuación (2).

$\mathrm{m}=\frac{\mathrm{y}_{2}-\mathrm{y}_{1}}{\mathrm{x}_{2}-\mathrm{x}_{1}}$

2)
A través de las curvas de nivel en la carta topográfica y aplicando el método de interpolación, se determinó la pendiente de las barrancas tomando lecturas cada 500 metros a lo largo del cauce. También se calculó el promedio aritmético para los puntos en cada barranca (SPIEGEL 2000) (Tablas 5 y 6 ).

Tabla 5. Barranca Colorada.

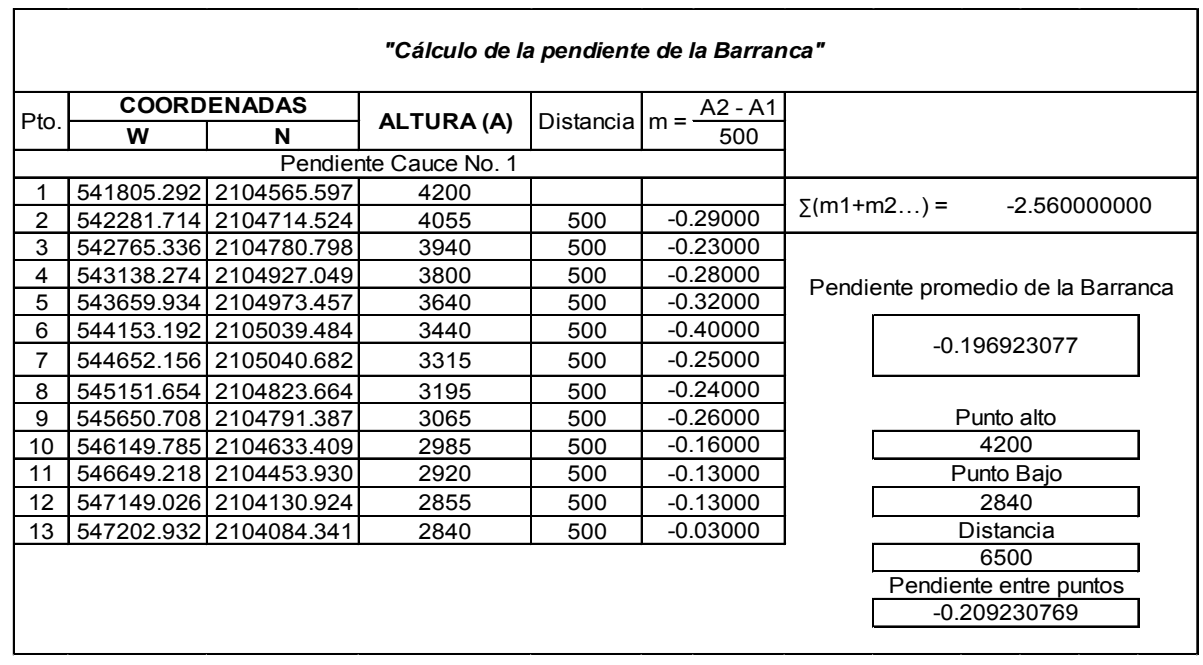

Tabla 6. Barranca Quimichule.

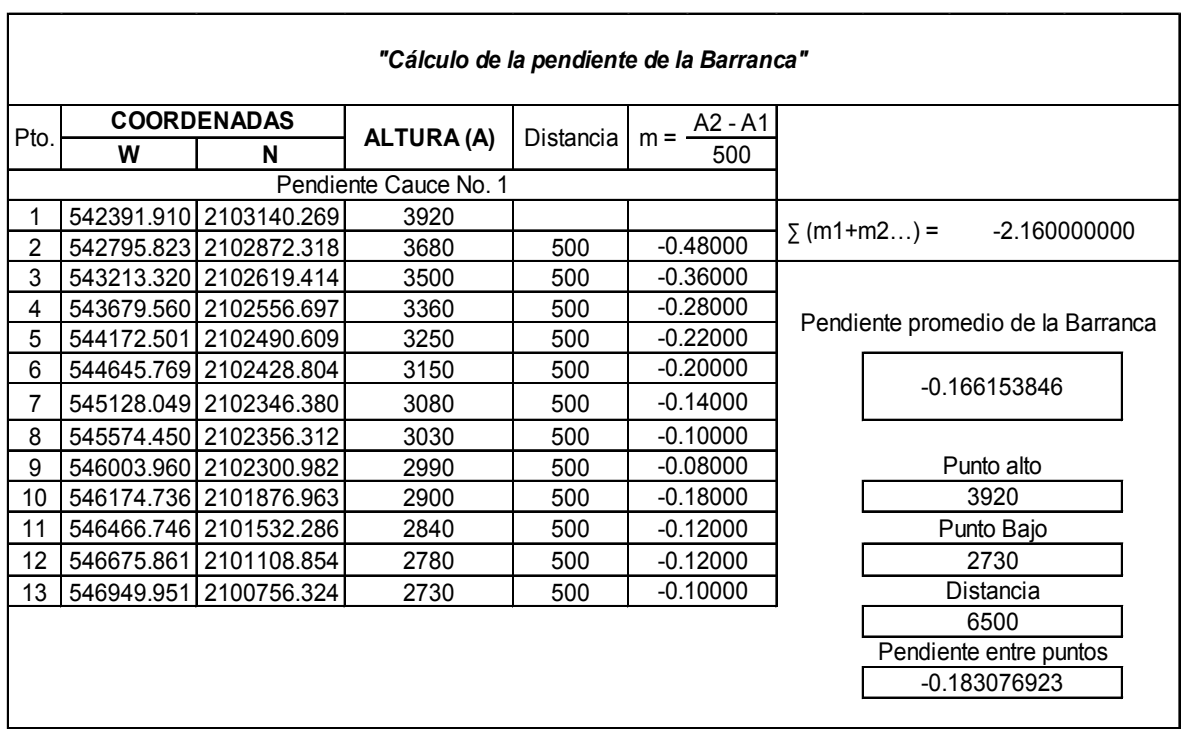




\section{RESULTADOS}

Análisis goeestadístico con el programa Geoeas

Utilizando el programa Geoeas, el cual genera gráficas de probabilidad normal ponderada de las variables forzantes (gravedad y altura), se obtuvo el comportamiento estadístico de dispersión con el modelo de regresión lineal de la matriz de variables analizadas, cuyo coeficiente de correlación para cada barranca procesada es cercano a-1 (fig. 4). Con una sobreposición del modelo surfer y la representación estadística desplegada, se observa la coincidencia entre los datos procesados y los modelados. También, se elaboraron gráficas que muestran el comportamiento de cada una de las variables consideradas (RAMOS et al. 2012). La estadística aplicada permitió interrelacionar perfiles de correlación entre varianzas, covarianzas, medias y rangos.

Se realizó el análisis geoestadístico de las barrancas, calculándose para cada una la desviación estándar que permite determinar el promedio aritmético de fluctuación de los datos respecto a su punto central o media, y la covarianza, medida de dispersión conjunta de dos variables estadísticas (RAMOS et al. 2010). Las medidas anteriores se utilizaron para obtener el coeficiente de correlación, cuyo resultado en general para cada barranca se aproxima a -1. Para efectos estadísticos se hizo un análisis con el fin de determinar la covarianza y el coeficiente de correlación para cada uno de los cauces y obtener sus gráficas correspondientes, tal como se presentan en las tablas 7 y 8 .

Barranca Colorada

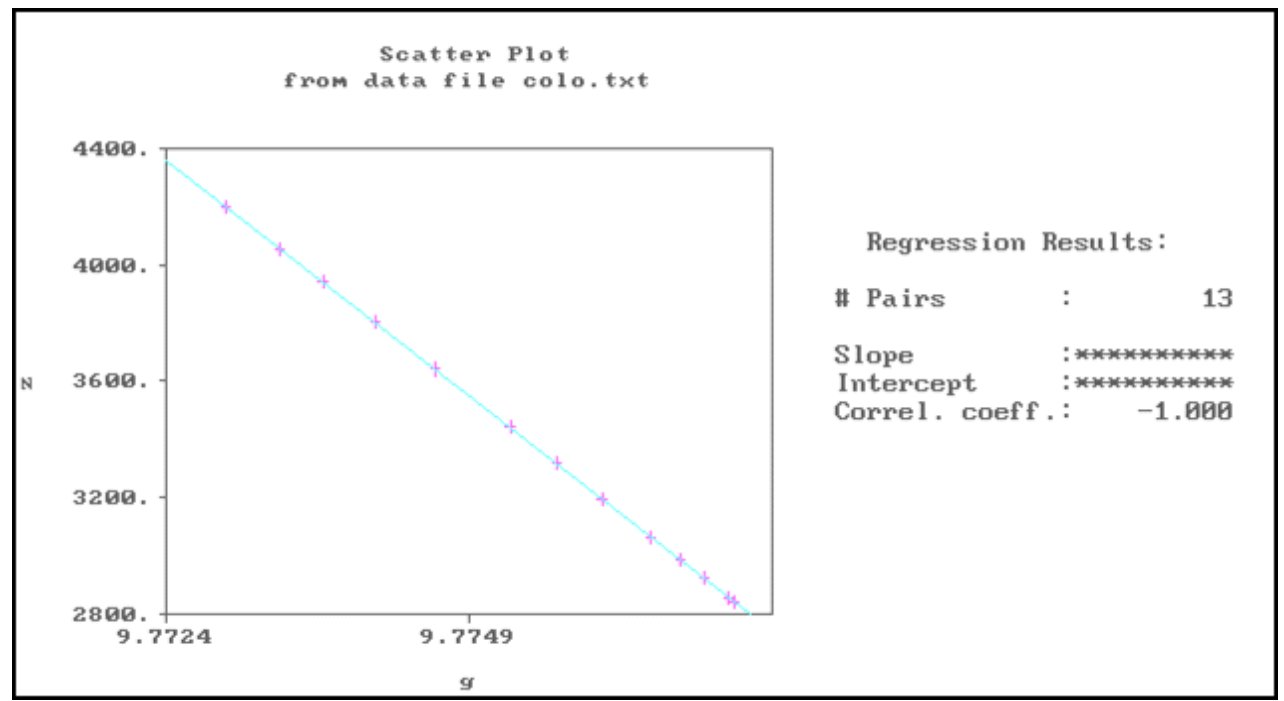

Fig. 4. Coeficiente de correlación para las barrancas Colorada y Quimichule.

Fig. 4. Correlation Coefficient for the Colorada and Quimichule streams. 
Barranca Quimichule

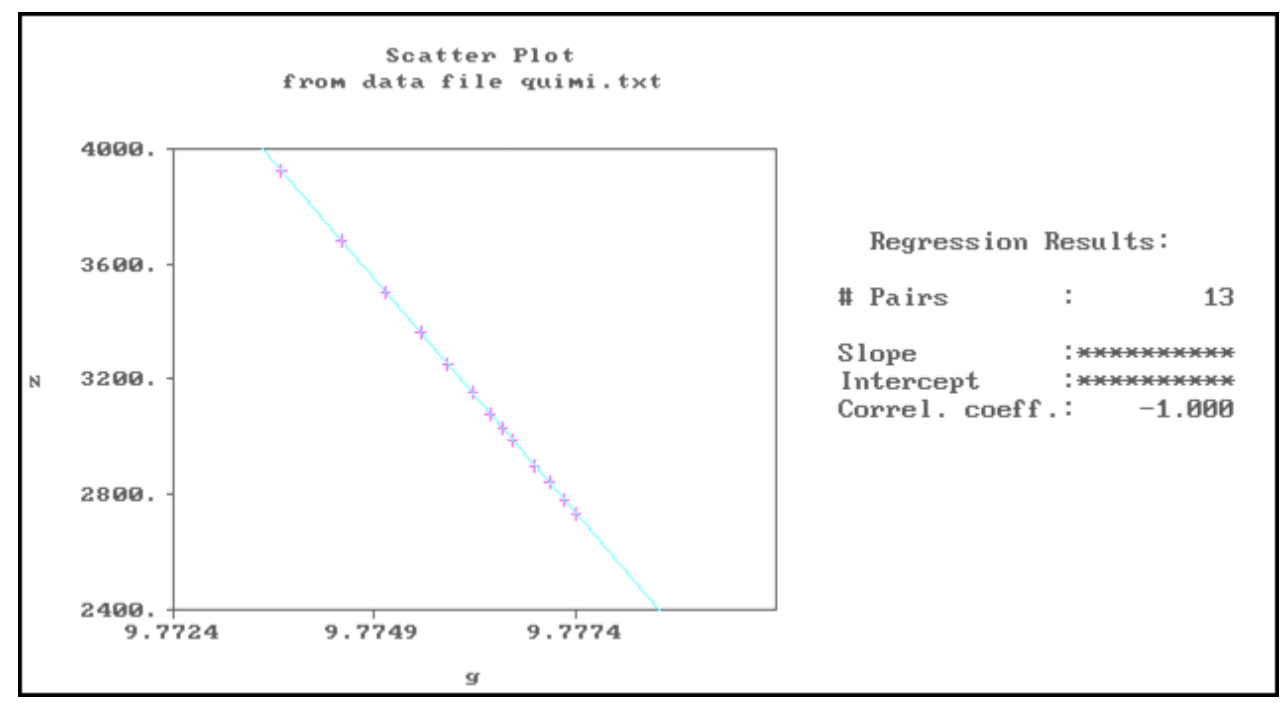

Fig. 4. Coeficiente de correlación para las barrancas Colorada y Quimichule.

Fig. 4. Correlation Coefficient for the Colorada and Quimichule streams.

Tabla 7. Barranca Colorada.

Table 7. Colorada Stream.

\begin{tabular}{|c|c|c|c|c|c|c|c|c|c|}
\hline Pto. & Altura (m) & & $\left(X_{i} \cdot \bar{X}\right)^{2}$ & & \begin{tabular}{|c|}
$\begin{array}{c}\text { Aceleración de la } \\
\text { gravedad local } \\
\left(\mathrm{m} / \mathrm{s}^{2}\right)\end{array}$ \\
\end{tabular} & & $\left(Y_{i} \cdot \bar{Y}\right)^{2}$ & & \\
\hline 1 & 4200 & 796,1538 & 633860,947 & & 9,772851 & $-0,002458$ & $6,03939 \mathrm{E}-06$ & & $-1,956560877$ \\
\hline 2 & 4055 & 651,1538 & 424001,331 & & 9,773300 & $-0,002009$ & $4,03731 \mathrm{E}-06$ & & $-1,308367578$ \\
\hline 3 & 3940 & 536,1538 & $287460,947 \mid$ & & 9,773655 & $-0,001654$ & $2,73601 \mathrm{E}-06$ & & $-0,886845767$ \\
\hline 4 & 3800 & 396,1538 & 156937,870 & & 9,774088 & $-0,001221$ & $1,49161 \mathrm{E}-06$ & & $-0,483828252$ \\
\hline 5 & 3640 & 236,1538 & 55768,639 & & 9,774582 & $-0,000727$ & $5,29011 \mathrm{E}-07$ & & $-0,171762177$ \\
\hline 6 & 3440 & 36,1538 & 1307,101 & & 9,775199 & $-0,000110$ & $1,20568 \mathrm{E}-08$ & & $-0,003969814$ \\
\hline 7 & 3315 & $-88,8462$ & 7893,639 & & 9,775585 & 0,000276 & $7,61465 \mathrm{E}-08$ & & $-0,024516798$ \\
\hline 8 & 3195 & $-208,8462$ & 43616,716 & & 9,775954 & 0,000645 & $4,16245 \mathrm{E}-07$ & & $-0,134741456$ \\
\hline 9 & 3065 & $-338,8462$ & 114816,716 & & 9,776355 & 0,001046 & $1,09449 \mathrm{E}-06$ & & $-0,354493546$ \\
\hline 10 & 2985 & $-418,8462$ & 175432,101 & & 9,776601 & 0,001292 & $1,66994 \mathrm{E}-06$ & & $-0,541259438$ \\
\hline 11 & 2920 & $-483,8462$ & 234107,101 & & 9,776801 & 0,001492 & $2,22589 \mathrm{E}-06$ & & $-0,721869941$ \\
\hline 12 & 2855 & $-548,8462$ & 301232,101 & & 9,777000 & 0,001691 & $2,85915 \mathrm{E}-06$ & & $-0,928044829$ \\
\hline \multirow[t]{2}{*}{13} & 2840 & $-563,8462$ & 317922,485 & \multirow{2}{*}{$\begin{array}{c}\text { Desviación Estándar } \\
460,2973698\end{array}$} & 9,777046 & 0,001737 & $3,01702 \mathrm{E}-06$ & \multirow{2}{*}{$\begin{array}{c}\text { Desviación Estándar } \\
0,001419758\end{array}$} & $-0,979377112$ \\
\hline & 3403,846 & & 211873,669 & & 9,775309 & & $2,01571 \mathrm{E}-06$ & & $-0,653510583$ \\
\hline & & & & & \multicolumn{3}{|c|}{$\begin{array}{c}\text { Coeficiente de Correlación } \\
-0,999999554\end{array}$} & & Covarianza \\
\hline
\end{tabular}




\section{Análisis geoestadístico con surfer}

Aplicando el programa Surfer se obtuvo el análisis de las variables estadísticas entre la altura y la gravedad (GASQUET 2000). Comparando los resultados de este análisis con las gráficas del Geoeas, se observa que el proceso de gravedad tiende a acercarse al valor calculado a medida que su altitud disminuye (Tablas 9 y 10).

Tabla 8. Barranca Quimichule.

Table 8. Quimichule STream.

"Estadística de la Barranca"

\begin{tabular}{|c|c|c|c|c|c|c|c|c|c|}
\hline Pto. & Altura (m) & & $\left(X_{i} \cdot \bar{X}\right)^{2}$ & & $\begin{array}{c}\text { Aceleración de la } \\
\text { gravedad local } \\
\left(\mathrm{m} / \mathrm{s}^{2}\right)\end{array}$ & & $\left(Y_{i} \cdot \bar{Y}\right)^{2}$ & & \\
\hline 1 & 3920 & 750 & 562500.0000 & & 9.773706 & -0.002312 & $5.34349 \mathrm{E}-06$ & & -1.733698657 \\
\hline 2 & 3680 & 510 & 260100.0000 & & 9.774448 & -0.001570 & $2.46559 \mathrm{E}-06$ & & -0.800811723 \\
\hline 3 & 3500 & 330 & 108900.0000 & & 9.775002 & -0.001016 & $1.03227 \mathrm{E}-06$ & & -0.335282639 \\
\hline 4 & 3360 & 190 & 36100.0000 & & 9.775434 & -0.000584 & $3.41395 \mathrm{E}-07$ & & -0.111015158 \\
\hline 5 & 3250 & 80 & 6400.0000 & & 9.775773 & -0.000245 & $6.0108 \mathrm{E}-08$ & & -0.019613542 \\
\hline 6 & 3150 & -20 & 400.0000 & & 9.776081 & 0.000063 & $3.98338 \mathrm{E}-09$ & & -0.00126228 \\
\hline 7 & 3080 & -90 & 8100.0000 & & 9.776297 & \begin{tabular}{|l|}
0.000279 \\
\end{tabular} & $7.76832 \mathrm{E}-08$ & & -0.025084539 \\
\hline 8 & 3030 & -140 & 19600.0000 & & 9.776451 & 0.000433 & $1.87542 \mathrm{E}-07$ & & -0.060628639 \\
\hline 9 & 2990 & -180 & 32400.0000 & & 9.776574 & 0.000556 & $3.09379 \mathrm{E}-07$ & & -0.100119271 \\
\hline 10 & 2900 & -270 & 72900.0000 & & 9.776850 & 0.000832 & $6.91932 \mathrm{E}-07$ & & -0.224592674 \\
\hline 11 & 2840 & -330 & 108900.0000 & & 9.777033 & \begin{tabular}{|l|l|}
0.001015 \\
\end{tabular} & $1.03074 \mathrm{E}-06$ & & -0.335033064 \\
\hline 12 & 2780 & -390 & 152100.0000 & & 9.777216 & 0.001198 & $1.43589 \mathrm{E}-06$ & & -0.467330943 \\
\hline \multirow[t]{2}{*}{13} & 2730 & -440 & 193600.0000 & \multirow{2}{*}{$\begin{array}{c}\text { Desviación Estándar } \\
346.6321482\end{array}$} & 9.777369 & 0.001351 & $1.8247 \mathrm{E}-06$ & \multirow{2}{*}{$\begin{array}{c}\text { Desviación Estándar } \\
0.001067156\end{array}$} & -0.594357709 \\
\hline & 3170 & & 120153.8462 & & 9.776018 & & $1.13882 \mathrm{E}-06$ & & -0.369910064 \\
\hline & & & & & \multicolumn{3}{|c|}{$\begin{array}{c}\text { Coeficiente de Correlación } \\
-0.999998549\end{array}$} & & Covarianza \\
\hline
\end{tabular}

Tabla 9. Barranca Colorada.

Table 9. Stream Colorada.

\section{Univariate Statistics}

\begin{tabular}{llll}
\hline & $X$ & $Y$ & $Z$ \\
\hline Minimum: & 2840 & 9.772851392 & 9.772851392 \\
25\%-tile: & 2985 & 9.774087594 & 9.774087594 \\
Median: & 3315 & 9.775584855 & 9.775584855 \\
$75 \%$-tile: & 3800 & 9.776601172 & 9.776601172 \\
Maximum: & 4200 & 9.777045867 & 9.777045867 \\
Midrange: & 3520 & 9.7749486295 & 9.7749486295 \\
Range: & 1360 & 0.0041944750000003 & 0.0041944750000003 \\
Interquartile Range: & 815 & 0.0025135780000003 & 0.0025135780000003 \\
Median Abs. Deviation: & 395 & 0.0012159950000008 & 0.0012159950000008 \\
& & & \\
Mean: & 3403.8461538462 & 9.7753089084615 & 9.7753089084615 \\
Trim Mean (10\%): & 3382.7272727273 & 9.7753744137273 & 9.7753744137273 \\
Standard Deviation: & 460.29736979376 & 0.0014197582068528 & 0.0014197582068528 \\
Variance: & 211873.66863905 & $2.015713365926 \mathrm{E}-0066$ & $2.015713365926 \mathrm{E}-0066$ \\
& & & \\
Coef. of Variation: & & & 0.00014523921649411 \\
Coef. of Skewness: & & & -0.34133729589633 \\
\hline
\end{tabular}


Tabla 10. Barranca Quimichule.

Table 10. Stream Quimichule.

\section{Univariate Statistics}

\begin{tabular}{llll}
\hline & $X$ & $Y$ & $Z$ \\
\hline Minimum: & 2730 & 9.773706207 & 9.773706207 \\
25\%-tile: & 2900 & 9.775433515 & 9.775433515 \\
Median: & 3080 & 9.776296522 & 9.776296522 \\
$75 \%$-tile: & 3360 & 9.77684963 & 9.77684963 \\
Maximum: & 3920 & 9.777368618 & 9.777368618 \\
Midrange: & 3325 & 9.7755374125 & 9.7755374125 \\
Range: & 1190 & 0.0036624110000005 & 0.0036624110000005 \\
Interquartile Range: & 460 & 0.0014161149999996 & 0.0014161149999996 \\
Median Abs. Deviation: & 240 & 0.00073653499999971 & 0.00073653499999971 \\
& & & \\
Mean: & 3170 & 9.7760178050769 & 9.7760178050769 \\
Trim Mean (10\%): & 3141.8181818182 & 9.7761051491818 & 9.7761051491818 \\
Standard Deviation: & 346.63214818283 & 0.0010671560113215 & 0.0010671560113215 \\
Variance: & 120153.84615385 & $1.1388219524997 \mathrm{E}-0061.1388219524997 \mathrm{E}-006$ \\
Coef. of Variation: & & & 0.00010916060430734 \\
Coef. of Skewness: & & & -0.72181350474623 \\
\hline
\end{tabular}

\section{Modelación estructural aplicando surfer}

La modelación estructural en 2D y 3D se realizó con el programa Surfer, lo que sirvió para identificar las barrancas de estudio y el comportamiento vectorial de las mismas (GODMAN 2001) (fig. 5 y 6).

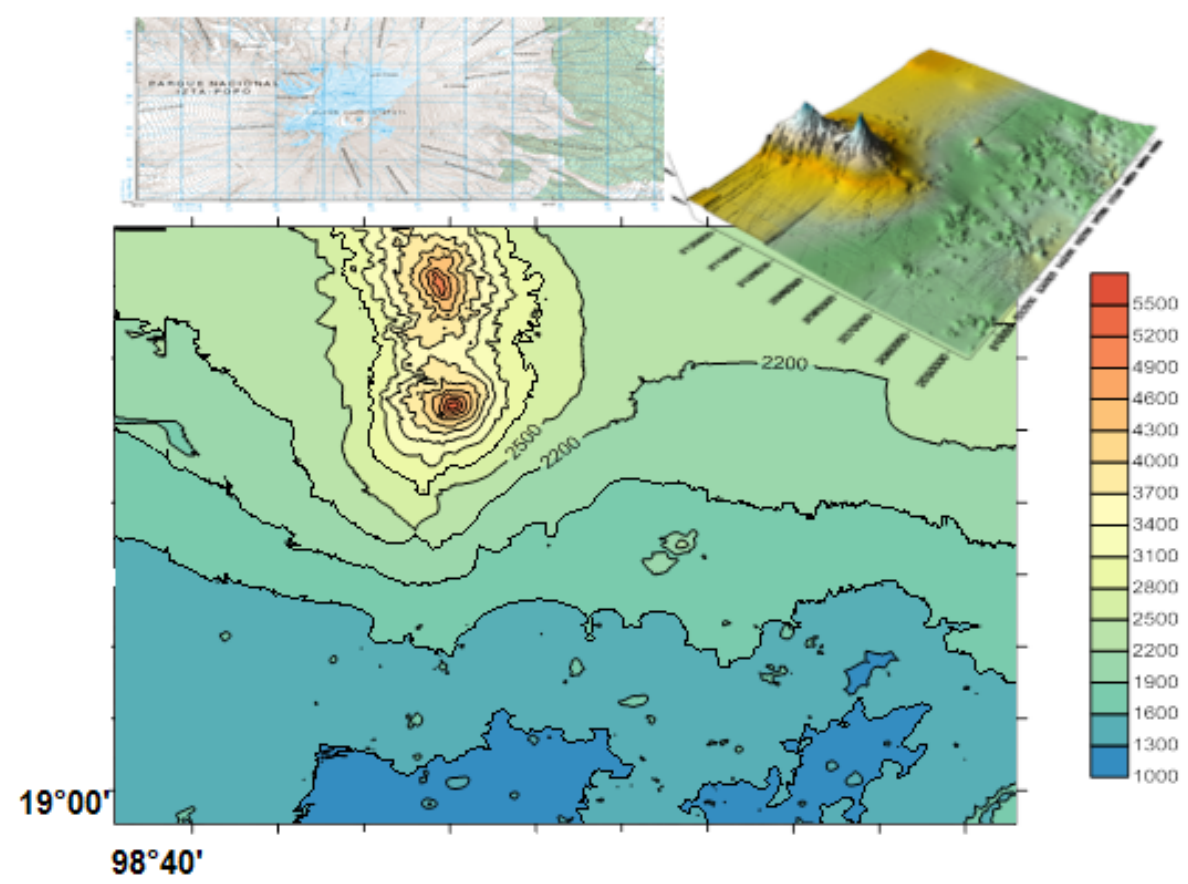

Fig. 5. Identificación de las barrancas en la zona del volcán Popocatépetl.

Fig. 5. identification streams in the volcano Popocatépetl area. 


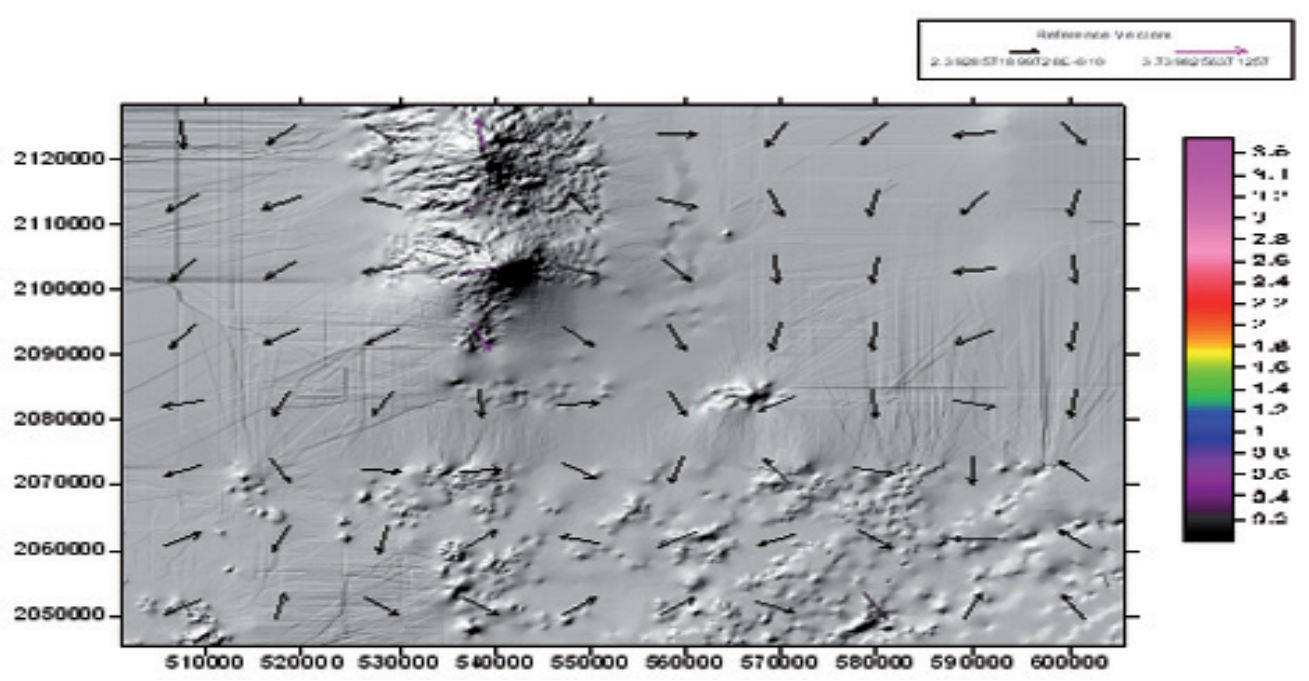

Fig. 6. Dirección vectorial de las barrancas con respecto a la gravedad.

Fig. 6. Stream vector orientation with respect to gravity.

El variograma es una herramienta que permite analizar el comportamiento espacial de una variable sobre un área definida (SCHENK
2002). En el caso de las barrancas, se elaboró el variograma de la altura contra la gravedad local calculada (Fig. 7).

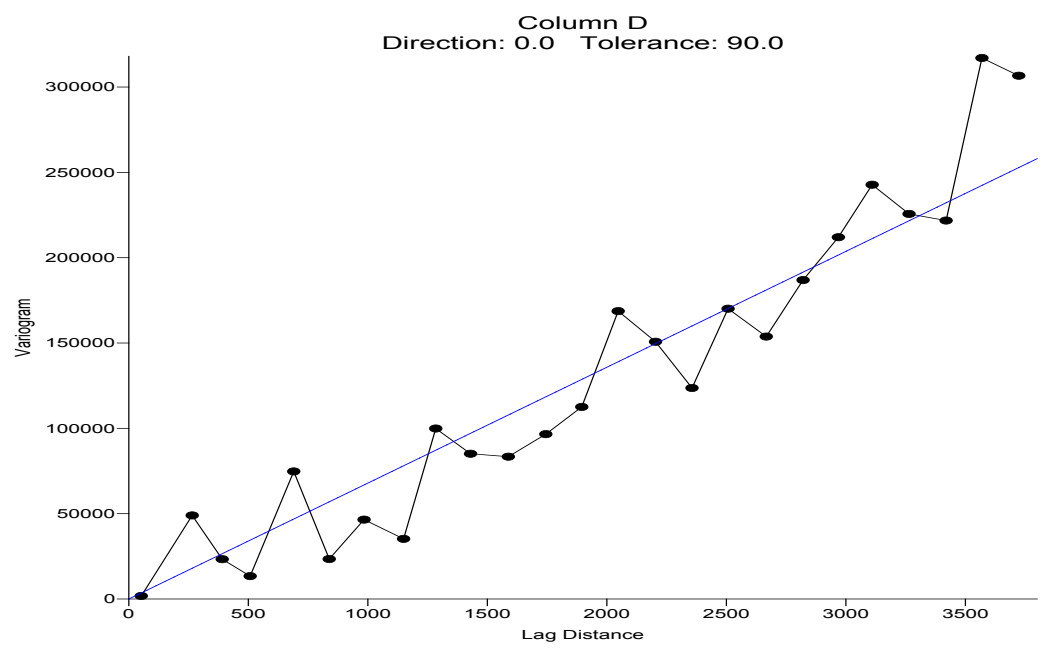

Fig. 7. Variograma de la altura respecto a la gravedad.

Fig. 7. Variogram height relative to gravity. 


\section{Cálculo de la fuerza del agua}

La fuerza del agua es la cantidad de empuje que ejerce este líquido en dirección de la pendiente; la densidad del agua es $1 \mathrm{gr} /$ $\mathrm{cm}^{3}$ o $1000 \mathrm{Kg} / \mathrm{m}^{3}$. Los cálculos realizados mediante el modelo matemático o ecuación mostraron que la fuerza aumenta proporcionalmente a la inclinación de la pendiente.

$\mathrm{F}_{1}=(\mathrm{d} * \cos \mathrm{m})(\mathrm{gl})$

El cauce del agua se subdividió con una equidistancia de 500 metros (LENNON 2002); los cálculos se muestran en las Tablas 11 y 12 .

Tabla 11. Barranca Colorada.

Table 11. Colorada Stream.

\begin{tabular}{|c|c|c|c|c|c|c|c|c|}
\hline \multicolumn{9}{|c|}{ "CÁLCULO DE LA FUERZA DEL AGUA" } \\
\hline \multirow{2}{*}{ Pto. } & \multirow{2}{*}{ Altura } & \multirow{2}{*}{$\begin{array}{c}\text { Densidad } \\
\text { del agua } \\
\left(\mathrm{Kg} / \mathrm{m}^{3}\right)\end{array}$} & \multirow{2}{*}{$m=\frac{A 2-A 1}{500}$} & \multirow{2}{*}{$\begin{array}{l}\text { Coseno de la } \\
\text { Pendiente } \\
\text { (Cos m) }\end{array}$} & \multirow{2}{*}{$\begin{array}{c}\text { Gravedad local } \\
\text { del punto }(\mathrm{gl})\end{array}$} & \multirow{2}{*}{$\begin{array}{l}\text { Fuerzas } \\
\text { parciales }\end{array}$} & \multirow{2}{*}{\multicolumn{2}{|c|}{$\mathrm{Fi}=\left(\mathrm{d}^{*} \cos \mathrm{m}\right)(\mathrm{gl})$}} \\
\hline & & & & & & & & \\
\hline \multirow{2}{*}{\multicolumn{7}{|c|}{ Pendiente del cauce }} & & \\
\hline & & & & & & & \multirow{2}{*}{\multicolumn{2}{|c|}{$\mathrm{SFi}=\quad 1173.05083838$}} \\
\hline 2 & 4055 & 1000 & -0.290000 & 0.999987 & 9.773300 & 97.731744 & & \\
\hline 3 & 3940 & 1000 & -0.230000 & 0.999992 & 9.773655 & 97.735761 & \multirow{3}{*}{\multicolumn{2}{|c|}{ Número de datos }} \\
\hline 4 & 3800 & 1000 & -0.280000 & 0.999988 & 9.774088 & 97.739709 & & \\
\hline 5 & 3640 & 1000 & -0.320000 & 0.999984 & 9.774582 & 97.744291 & & \\
\hline 6 & 3440 & 1000 & -0.400000 & 0.999976 & 9.775199 & 97.749609 & \multirow{2}{*}{\multicolumn{2}{|c|}{12}} \\
\hline 7 & 3315 & 1000 & -0.250000 & 0.999990 & 9.775585 & 97.754918 & & \\
\hline 8 & 3195 & 1000 & -0.240000 & 0.999991 & 9.775954 & 97.758683 & \multirow{4}{*}{\multicolumn{2}{|c|}{$F=\sum_{i=1}^{n} F_{i}$}} \\
\hline 9 & 3065 & 1000 & -0.260000 & 0.999990 & 9.776355 & 97.762544 & & \\
\hline 10 & 2985 & 1000 & -0.160000 & 0.999996 & 9.776601 & 97.765631 & & \\
\hline 11 & 2920 & 1000 & -0.130000 & 0.999997 & 9.776801 & 97.767757 & & \\
\hline 12 & 2855 & 1000 & -0.130000 & 0.999997 & 9.777000 & 97.769746 & \multirow{2}{*}{\multicolumn{2}{|c|}{$97.75423653 \mathrm{~N}$}} \\
\hline 13 & 2840 & 1000 & -0.030000 & 1.000000 & 9.777046 & 97.770445 & & \\
\hline
\end{tabular}

Tabla 12. Barranca Quimichule.

Table 12. Quimichule Stream

\begin{tabular}{|c|c|c|c|c|c|c|c|}
\hline \multicolumn{8}{|c|}{ "CÁLCULO DE LA FUERZA DEL AGUA" } \\
\hline \multirow{2}{*}{ Pto. } & \multirow{2}{*}{ Altura } & \multirow{2}{*}{$\begin{array}{c}\text { Densidad } \\
\text { del agua } \\
\left(\mathrm{Kg} / \mathrm{m}^{3}\right)\end{array}$} & \multirow{2}{*}{$m=\frac{A 2-A 1}{500}$} & \multirow{2}{*}{\begin{tabular}{|c|} 
Coseno de la \\
Pendiente (Cos \\
m)
\end{tabular}} & \multirow{2}{*}{$\begin{array}{l}\text { Gravedad local } \\
\text { del punto ( } \mathrm{gl} \text { ) }\end{array}$} & \multirow{2}{*}{$\begin{array}{l}\text { Fuerzas } \\
\text { Parciales }\end{array}$} & \multirow[b]{2}{*}{$\mathrm{F} \mathrm{i}=\left(\mathrm{d}^{*} \cos \mathrm{m}\right)(\mathrm{gl})$} \\
\hline & & & & & & & \\
\hline \multicolumn{7}{|c|}{ Pendiente del cauce } & \multirow{3}{*}{$\mathrm{SFi}=\quad 1173.1358132$} \\
\hline 1 & 3920 & & & & & & \\
\hline 2 & 3680 & 1000 & -0.480000 & 0.999965 & 9.774448 & 97.741046 & \\
\hline 3 & 3500 & 1000 & -0.360000 & 0.999980 & 9.775002 & 97.748088 & \multirow{3}{*}{ Número de datos } \\
\hline 4 & 3360 & 1000 & -0.280000 & 0.999988 & 9.775434 & 97.753168 & \\
\hline 5 & 3250 & 1000 & -0.220000 & 0.999993 & 9.775773 & 97.757006 & \\
\hline 6 & 3150 & 1000 & -0.200000 & 0.999994 & 9.776081 & 97.760214 & \multirow{2}{*}{12} \\
\hline 7 & 3080 & 1000 & -0.140000 & 0.999997 & 9.776297 & 97.762673 & \\
\hline 8 & 3030 & 1000 & -0.100000 & 0.999998 & 9.776451 & 97.764360 & \multirow{4}{*}{$F=\sum_{i=1}^{n} F_{i}$} \\
\hline 9 & 2990 & 1000 & -0.080000 & 0.999999 & 9.776574 & 97.765645 & \\
\hline 10 & 2900 & 1000 & -0.180000 & 0.999995 & 9.776850 & 97.768014 & \\
\hline 11 & 2840 & 1000 & -0.120000 & 0.999998 & 9.777033 & 97.770116 & \\
\hline 12 & 2780 & 1000 & -0.120000 & 0.999998 & 9.777216 & 97.771946 & \multirow{2}{*}{$97.76131777 \mathrm{~N}$} \\
\hline 13 & 2730 & 1000 & -0.100000 & 0.999998 & 9.777369 & 97.773537 & \\
\hline
\end{tabular}




\section{Cálculo del gasto}

El gasto es el volumen de un líquido que pasa por una sección en un determinado tiempo. Para este caso se obtuvo el gasto de cada una de las barrancas aplicando la ecuación (4) (Tablas 13 y 14).
Donde:

$Q=$ Gasto en $\mathrm{m}^{3} / \mathrm{s}$

$k=$ coeficiente de permeabilidad

$i=$ Gradiente hidráulico

$A=$ Área de captación

$Q=0.278 * k * i * A$

Tabla 13. Barranca Colorada.

Table 13. Stream Barranca.

\begin{tabular}{|l|c|}
\hline \multicolumn{2}{|c|}{ "Cálculo del gasto" } \\
\hline Área de la cuenca hidrológica & $4324587 \mathrm{~m}^{2}$ \\
\hline Longitud del cauce & $6500 \mathrm{~m}$ \\
\hline Coeficiente de permeabilidad (k) & $0,05 \mathrm{~cm} / \mathrm{s}$ \\
\hline Gradiente hidráulico (i) & $0,183077 \mathrm{~m}$ \\
\hline \multicolumn{1}{|c|}{ Gasto } & $110,050759 \mathrm{~m}^{3} / \mathrm{s}$ \\
\hline
\end{tabular}

Tabla 14. Barranca Quimichule.

Table 14. Quimichule Stream.

\begin{tabular}{|c|c|}
\hline \multicolumn{2}{|c|}{ "Cálculo del gasto" } \\
\hline Área de la cuenca hidrológica & $2356300 \mathrm{~m}^{2}$ \\
\hline Longitud del cauce & $6500 \mathrm{~m}$ \\
\hline Coeficiente de permeabilidad (k) & $0.05 \mathrm{~cm} / \mathrm{s}$ \\
\hline Gradiente hidráulico (i) & $0.209231 \mathrm{~m}$ \\
\hline Gasto & $68.528454 \mathrm{~m}^{3} / \mathrm{s}$ \\
\hline
\end{tabular}

\section{DISCUSIÓN Y CONCLUSIONES}

Es importante destacar que dentro de la literatura relacionada con investigaciones del volcán Popocatépetl, no se ha encontrado un análisis similar al presente. El trabajo muestra los resultados obtenidos del procesamiento de datos cartográficos y la aplicación de los programas Geoeas y Surfer para calcular la relación entre la aceleración local de la gravedad, la altura, la pendiente, el gasto y la fuerza de un líquido que corre sobre un perfil de las barrancas Colorada y Quimichule.
La gravedad, la pendiente, la fuerza y el gasto son menores en la barranca Colorada, resultados que validan los métodos aplicados. El coeficiente de correlación entre la gravedad y la altura muestra que existe una correlación negativa perfecta, es decir, a mayor altura menor gravedad.

El valor absoluto del caudal obtenido en cada barranca no es comparable, ya que la superficie de la barranca Colorada es solamente del $54.5 \%$ de la barranca Quimichule, y para poder compararlo es necesario obtener el caudal específico de 
cada una (dividiendo su superficie, que habitualmente se proporciona en $\mathrm{m}^{3} / \mathrm{s}$ $\mathrm{km}^{2}$ o en litros $/ \mathrm{s} \mathrm{km}^{2}$ ). Así entonces, los caudales específicos de cada barranca serían:

Colorada $\left(68.528454 \mathrm{~m}^{3} / 2.3563 \mathrm{~s} \mathrm{~km}^{2}\right)$ $=0.2908311 \mathrm{~m}^{3} / \mathrm{s} \mathrm{km}^{2}$ o $290.83111 / \mathrm{s} \mathrm{km}^{2}$

Quimichule (110.050759 $\mathrm{m}^{3} / 4.324587 \mathrm{~s}$ $\left.\mathrm{km}^{2}\right)=0.254477 \mathrm{~m}^{3} / \mathrm{s} \mathrm{km}{ }^{2}$ o $254.477 \mathrm{l}$ $/ \mathrm{s} \mathrm{km}^{2}$

De tal manera que la barranca Quimichule produce por cada $\mathrm{km}^{2}$ un $87.5 \%$ del agua que escurre por la barranca Colorada, siendo esta última la que mostraría una capacidad más elevada de generar escorrentía.

Este resultado se puede comparar con la gravedad local de $9.7760 \mathrm{~m} / \mathrm{s}^{2}$ en Quimichule y $9.7753 \mathrm{~m} / \mathrm{s}^{2}$ en Colorada con los valores de fuerza del agua de $97.76131777 \mathrm{~N}$ en Quimichule $\mathrm{y}$ de $97.75423653 \mathrm{~N}$ en Colorada, con los valores de la pendiente promedio de -0.166153846 en Quimichule y -0.196923077 en Colorada. Se revela local y que las pequeñas diferencias de gravedad $(0.0007 \%$ mayor en Quimichule) ejercen una influencia equivalente sobre la fuerza del agua, lo que hace suponer que se debe básicamente a las diferencias de altitud que muestran ambas barrancas. Sin embargo, estas variables no intervienen en el cálculo de los caudales (Q), donde la diferencia de pendiente media o gradiente hidráulico (i) son determinantes en el cálculo teórico de caudales; la misma diferencia observada entre los valores de i se manifiesta entre los valores de Q (87.5\% entre Quimichule y Colorada).

La aplicación de modelos geoestadísticos pone de manifiesto la importancia de la aplicación matemática en los análisis geomorfológicos, presentando diferentes gráficas y datos comparativos de los análisis, así como los estudios de modelados estructurales en procesos geomorfológicos e hidrológicos, que son útiles para tener una idea del comportamiento de flujos de agua, de lodo o de magma, ocasionados por las lluvias o la actividad del Popocatépetl y que podrían afectar a las poblaciones cercanas a las barrancas.

Este estudio pretende contribuir a los trabajos ya existentes, y se considera que los resultados obtenidos a través de herramientas tecnológicas representan un valioso aporte para el área de prevención de desastres naturales. Se insiste que la aplicación de modelos diferentes con certeza llevaría a obtener resultados similares.

\section{REFERENCIAS}

GARCÍA, F., R. RAMOS \& R. DOMÍNGUEZ, 1996. Posible flujo de lodo en el costado oriente del volcán Popocatépetl. En: Volcán Popocatépetl, Estudios Realizados Durante la Crisis de 1994-1995, Sistema Nacional de Protección Civil, Centro Nacional de Prevención de Desastres, Universidad Nacional Autónoma de México, México: 109-125.

FRANCO, J. M., A. M. CASSANO \& G.L. BOLLA, 2005. Estabilidad de barrancas sobre el río Paraná. Departamento de Ingeniería Civil de la Universidad Regional de Paraná. 1-119.

SPIEGEL, M., 2000. Probabilidad y Estadística. Ed. Mc. Graw Hill. México.

RAMOS, R., P. MÁXIMO,A. MONTIEL, Y. GONZÁLEZ \& A. RODRÍGUEZ, 2010. Análisis fotogramétrico del volcán Citlaltépetl. Cartográfica, 84:105-116. 
RAMOS, R., P. MÁXIMO, J. NARCISO, M. MIRÓN \& M. BELTRÁN, 2012. Estudio geoestadístico para obtener la gravedad local, pendiente y cálculo hidrológico de las barrancas Xaltelulco, Tepeloncocone y Tenepanco del volcán Popocatépetl. Boletín de Ciencias de la Tierra, 31: 65-84.

GASQUET, C., 2000. Analyse de fourier et applications. Ed. Dunod. France.
GODMAN, P., 2001. Principles of geographical information systems For Land Resources. Springer. USA.

SCHENK, T., 2002. Fotogrametría digital. Ed. Marcombo-ICC. España.

LENNON, T., 2002. Remote sensing digital image analysis, Esa/Esrin. USA.

\section{Imagen de Investigaciones GEOGRÁFICAS}

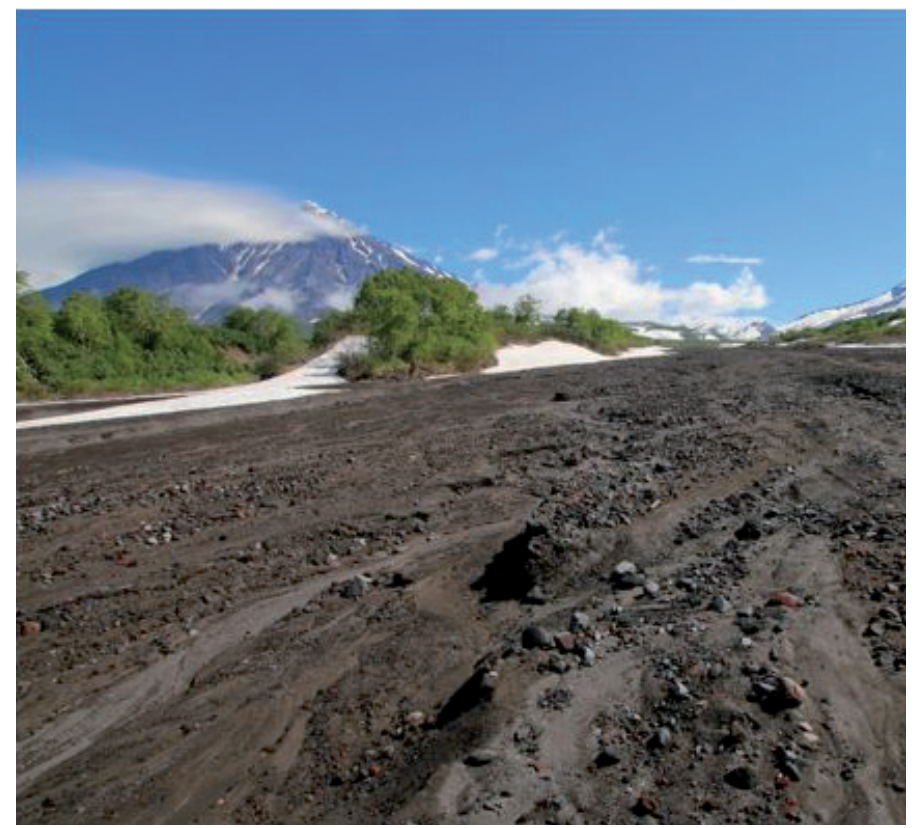

Lahar deposits in the Avacha catchment close to Pietropavlovsk, Southern Kamtschatka, Russia. In the background the Koryaksky Volcano (3456 m) on the left and the Avacha Volcano (2741 m) on the right.

EU-IRSES Project entitled: "Fluvial processes and sediment dynamics of slope channel systems: Impacts of socio economic-and climate change on river system characteristics and related services (FLUMEN)"

The FLUMEN project is an EU funded scientific exchange programme. It is focused on the ecological and morphological characteristics of river channels and related processes of erosion, sediment transport and deposition under changing boundary conditions, described by socio economic and climate change scenarios. We study effects of these changes on river systems at different spatial and temporal scales. The specific focus of the project is addressing the interface between the river channel and related slope systems. We study also the historic evolution of river systems and simulate future developments using scenarios.

Dr. Michael Maerker. Earth Sciences Department, University of Florence, Italy; Heidelberg Academy of Sciences and Humanities, Germany. mmaerker@unifi.it/ www.flumen-irses.eu 